\title{
summaries
}

\section{Oral health promotion can be effective in changing knowledge}

\section{Sprod A, Anderson R, Treasure E T. Effective Oral Health Promotion. Literature Review. Health Promotion Wales. Technical Report 201996}

Objective To identify oral health promotion practices which have been shown to be effective or ineffective. To highlight practices which need further research and evaluation.

Data sources Studies were identified from Medline, printed indices, personal contact and from reference lists of retrieved articles for the period 1982 to January 1996.

Study selection Papers whose aims were to evaluate oral health promotion or describe policy and practice in Oral Health Promotion. Papers relating to other health promotional activities e.g. diet and nutrition were not included unless specific oral health indicators were used for evaluation. All selected studies were assessed according to 9 quality criteria for study design and 3 criteria for value of findings for oral health gain. A random selection of the papers was assessed independently by the three researches to ensure consistency. Differences were discussed and resolved.

\begin{abstract}
Main findings 230 papers were initially identified 113 not fitting the review criteria were excluded. 18 papers with strong study design were identified including 3 RCTs. The report describes in some detail a wide selection of identified papers and also includes a list where papers are identified as having evidence of effectiveness, unclear evidence or evidence of ineffectiveness. A table allows identification of studies carried out by location and provider.
\end{abstract}

Conclusions The review reaches 17 different conclusions among which are; there is clear evidence that oral health education/promotion can be effective in bringing about changes in people's knowledge. It is unclear whether one-off oral health promotion initiatives are sufficient to improve individuals' oral health significantly for long periods. Water fluoridation is effective at preventing dental caries.

Address A. Sprod, Dental Public Health Unit, University of Wales College of Medicine, Heath Park, Cardiff, UK

\section{Commentary}

Within the space of a year, two major reviews of the effectiveness of oral health promotion were published by Health Promotion Wales (HPW) in 1996 and the Health Education Authority (HEA) in 1997. They fulfil a major need for evidence of effectiveness in this area of practice and hopefully are being widely read by practitioners in oral health promotion as well as the dental professions, in general. In assessing both reviews, three broad issues need to be considered; Are the results valid? What are the results? Will the results help locally?

In terms of validity, both set out in some detail the research strategies and quality criteria for inclusion. It is an area where review methodologies are being continuously refined and the timing of the two reports may reflect some of these changes. The HPW report confines its searches principally to the Medline Electronic Database, whereas the HEA covers a wider group of databases as well as including other areas such as the grey literature. It would be interesting to do a full comparison of the retrieval rates for both approaches. From a small sample of five papers on randomised controlled trials for caries, the HPW approach detected three out of five eligible papers in the NBA document. The use of statistics and analysis was attempted in the HEA paper but was specifically excluded from the approach by HPW. This is an area not without difficulty as highlighted in Richard Watts' foreword to the HEA document concerning the social science base of health promotion.

The results of both reviews underline the difficulties in finding good quality research evidence of effectiveness in this area of practice, either because of poor study design or because the outcomes of intervention are difficult to measure. The main findings of the reviews are:

\section{Health Promotion (Wales)}

- Oral health promotion can be effective in bringing about changes in people knowledge and in improving health.

- Limited behavioural change could be achieved using simple approaches and longer term changes using more tailored approaches.

- Health education which targets whole populations may increase inequalities in health.

- Use of fluoride in water supplies, toothpaste and supplements is effective. No measures will achieve the same level of prevention as fluoridation, for the same resources.

\section{Health Education Authority}

- Oral health promotion, including the use of fluoride, is effective, but there is no evidence of effectiveness of education programmes aimed at caries reduction, which do not use fluoride.

- School based health education aimed at improving oral hygiene has not shown to be effective but clinical chairside, oral hygiene interventions have been shown to be effective.

- Simple oral health promotion approaches are as effective as more complex interventions in reducing plaque levels, but the changes are difficult to sustain.

- Oral health promotion is effective for increasing knowledge but there is no evidence that changes in knowledge result in changes in behaviour.

There is no convincing evidence of effectiveness of mass media programmes designed to promote oral health.

It is interesting to compare not only 